Aim of the study was to compare radiobiological effects of multiple vs. single low-dose pre-irradiation on the HT29 cell line. This regime is designed to be as similar as possible to fractionated tumour radiotherapy treatment and to provide data on radiobiological effects on human tumour cells.

Material and methods: The cell line used in the study was HT29 (human colorectal adenocarcinoma, American Type Culture Collection HTB- $38^{\text {тM }}$ ). Also, for comparison, the MRC5 cell line (human foetal lung fibroblasts, American Type Culture Collection CCL 171) was used.

Four-day treatment in a $4 \times 2$ Gy regime was performed. Cell viability was evaluated by tetrazolium colorimetric MTT assay.

Results: Multiple low-dose pre-irradiation induced a stronger radioadaptive response compared to single low-dose application in the HT29 cell line. Multiple pre-irradiation with 0.03 Gy and 0.05 Gy caused radioadaptive effects, while in both single and multiple lowdose pre-irradiation regimes $0.07 \mathrm{~Gy}$ led to radiosensitivity. Radiobiological effects induced in the HT29 cell line by low-dose pre-irradiation were evidently weak during the treatment time because a single low-dose applied only on the first day gave no radioadaptive effects. In the MRC5 cell line different effects were registered, since radioadaptive response has not been observed after multiple or single pre-irradiation. Conclusions: The obtained data are interesting, especially for the possible application of low-dose pre-irradiation in radiotherapy.

Key words: HT29 cell line, low-dose irradiation, radioadaptive response, radiosensitivity.

Contemp Oncol (Pozn) 2014; 18 (4): 230-233 DOI: $10.5114 /$ wo.2014.41386

\section{Radiobiological effects of multiple vs. single low-dose pre-irradiation on the HT29 cell line}

Igor Djan ${ }^{1,3}$, Slavica Solajic ${ }^{1}$, Mihajla Djan², Natasa Vucinic ${ }^{3}$, Dunja Popovic ${ }^{2}$, Miroslav Ilic $^{3,4}$, Silvija Lučić, ${ }^{1,3}$, Gordana Bogdanovic ${ }^{1,3}$

${ }^{1}$ Institute of Oncology Vojvodina, Sremska Kamenica, Serbia

${ }^{2}$ Faculty of Sciences, University of Novi Sad, Novi Sad, Serbia

${ }^{3}$ Medical Faculty, University of Novi Sad, Serbia

${ }^{4}$ Institute of Pulmonary Diseases Vojvodina, Sremska Kamenica, Serbia

\section{Introduction}

Radiobiology studies the effects of electromagnetic radiation on biological systems. These effects may include DNA damage, point mutations, chromosome aberrations, cell killing, disturbances in cell cycle and cell proliferation etc. [1]. Radiobiological effects depend on irradiation dose [2]. Very low-dose irradiation has different effects on cells in vitro, compared with high doses. Cell and tissue exposure to low doses followed by higher irradiation doses is known as radioadaptive irradiation. An adaptive dose is a low dose that can be applied before one or multifraction challenging doses. Adaptive response can lead to hypersensitivity or radioresistance [3]. It is known that low-dose irradiation can lead to two phenomena: to the bystander effect and to radioresistance or radiosensitivity [2].

Previous in vitro and in vivo experiments using low-dose irradiation followed by high doses showed a reduction in the level of apoptosis associated with radioadaptive response [4, 5]. Radioadaptive response is registered using an ionising radiation dose below 0.1 Gy. Radioadaptive response can be examined by cell viability tests, chromosome aberration detection and tracking DNA breaks and repair systems [6]. Based on functional and single gene investigations, it has been suggested that the adaptive response phenotype is associated with DNA damage repair and stress response functions [7]. Changes of these functions may lead to the reduction of cytogenetic damages, and thus enhance the survival rate in mammalian cells [8-10].

The adaptive response could be used in particular radiotherapy indications. Several studies reported on the effects of pre-irradiation doses and variable challenging doses, but the exact mechanism of the effects is still unknown [7, 11, 12]. Cell viability is a widely used parameter for cell line or model organism survival evaluation [13]. Previous studies [14, 15] on low-dose irradiation effects on cell viability were performed using low doses followed by single high-dose irradiation. Schwarz et al. [13] showed an adaptive effect in HT29 and GM637 cell lines suggesting that 0.05 Gy might be the dose level that increases radiosensitivity of tumour cells whilst sparing the effect on the viability of normal cells.

Our aim was to compare radiobiological effects of multiple vs. single low doses pre-irradiation on the HT29 cell line in four-day treatment in a 4 × 2 Gy regime. This regime is designed in order to mimic tumour radiotherapy treatment, which could be useful in providing data on radiobiological effects on human tumour cells.

\section{Material and methods}

Cell line

The cell line used in the study was HT29 (human colorectal adenocarcinoma, American Type Culture Collection HTB-38 ${ }^{\mathrm{TM}}$ ). Also, for the compar- 
ison and evaluation of the data, MRC5 cell line (human foetal lung fibroblasts, American Type Culture Collection CCL 171) was used. The cells were grown in Dulbecco's modified Eagle's medium (DMEM) with $4.5 \%$ of glucose, supplemented with $10 \%$ of foetal calf serum (FCS, NIVNS) and antibiotics: $100 \mathrm{IU} / \mathrm{cm}^{3}$ of penicillin and $100 \mathrm{\mu g} / \mathrm{cm}^{3}$ of streptomycin (ICN Galenika). The cells were sub-cultured twice a week and a single cell suspension was obtained using $0.25 \%$ trypsin in EDTA (Serva). All cell lines were cultured in flasks (Costar, $25 \mathrm{~cm}^{2}$ ) at $37^{\circ} \mathrm{C}$ in a $100 \%$-humidity atmosphere and $5 \% \mathrm{CO}_{2}$. Exponentially growing cells were used throughout the assays. The cell density (number of cells per unit volume) and percentage of viable cells were performed as previously described [16]. The viability of the cells used in the assay was over $90 \%$.

\section{Irradiation}

Cell line was irradiated using a phantom constructed for our experimental requirements following these principles: to minimise the presence of air between the flask and the hole where the flask is inserted, and therefore improve scatter conditions in the medium and allow isodose coverage of $95-107 \%$. The phantom was made of Plexiglas plates, due to its tissue equivalent characteristics. There were four holes to insert the flasks (Sarstedt, $25 \mathrm{~cm}^{2}$ ). The size of the phantom $(30 \mathrm{~cm} \times 30 \mathrm{~cm} \times 5 \mathrm{~cm})$ allowed sufficient scatter material around the radiation field to cover the flasks in all three directions. Flasks with cell lines were placed into phantom holes and were CT scanned. The CT data were imported into a treatment planning system, contoured and planned with the commercial treatment planning system, Elekta XiO, version 4.62. The cell samples were pre-irradiated with 0.03 Gy, 0.05 Gy and 0.07 Gy. There were two types of control samples: non-irradiated cells (I) and non-pre-irradiated cells (II). Control II and pre-irradiated cells were further irradiated two hours after pre-irradiation, when the regime of 2 Gy daily, during four days, was applied. The first group of samples was pre-irradiated only once (single low dose), on the first day of treatment, and the second group was pre-irradiated every day (multiple low doses) during the four-day treatment. The described regimes of irradiation were repeated twice. The cell lines were irradiated using Varian 600DBX; 6 × MV photon energy was used. For low dose pre-irradiation of 0.03, 0.05 and $0.07 \mathrm{~Gy}$, an 80-MU/min dose rate was applied and for the 2 Gy pre-irradiation, a $400-\mathrm{MU} / \mathrm{min}$ dose rate was used.

\section{Colorimetric MTT (tetrazolium) assay}

Cell viability was evaluated by tetrazolium colorimetric MTT assay (SIGMA). The assay is based on the cleavage of the tetrazolium salt MTT (3-(4,5-dimethylthiazol-2-yl)-2,5-diphenyl tetrazolium bromide), into formazan by mitochondrial dehydrogenases in viable cells [17]. MTT (5 mg/ml) was dissolved in phosphate buffered saline (PBS) and filtered to sterilise and remove the small amount of insoluble residue present in some batches of MTT. Stock MTT solution (10 $\mu \mathrm{l}$ per $100 \mu \mathrm{l}$ medium) was added to all wells, and plates were incubated at $37^{\circ} \mathrm{C}$ for
4 hours. Acid isopropanol $(100 \mu \mathrm{l}$ of $0.04 \mathrm{~N} \mathrm{HCl}$ in isopropanol) was added to all wells and mixed thoroughly to dissolve the dark blue crystals. After a few minutes at room temperature to ensure that all crystals were dissolved, the plates were read on a spectrophotometer microplate reader (Multiscan MCC340, LabSystems) at 540/690 nm. Plates were normally read within 1 hour of adding the isopropanol. The wells without cells containing complete medium and MTT only acted as blanks.

\section{Data analysis}

All continuous variables are expressed as means \pm standard deviation (SD). One-way variance analysis (ANOVA) was applied to test the differences in cell survival among controls and the different pre-irradiation doses applied, and Duncan and Tuckey post-hoc tests were performed after detecting the significance [18]. A probability value less than 0.05 was regarded as statistically significant.

\section{Results}

Radiobiological effects of multiple vs. single low-dose pre-irradiation were presented as absorbance values obtained spectrophotometrically, which correlates to the number of viable and metabolically active cells (Figs. 1 and 2). It is clear that radiobiological effects differ depending on the applied irradiation regime.

Compared to non-irradiated control (I), a significant decrease of cell viability in the HT29 cell line was found after each, single pre-irradiation dose applied $(p<0.05$, Duncan test). A single pre-irradiation dose of 0.03 Gy gave a non-significant increase, while 0.05 Gy and 0.07 Gy led to non-significant decreases in cell viability compared to the control (II) without pre-irradiation (Fig. 1).

A statistically significant difference in cell survival was found between $0.03 \mathrm{~Gy}$ and 0.05 Gy pre-irradiation, and between 0.03 Gy and 0.07 Gy, respectively, only for the single, low-dose pre-irradiation regime $(p<0.05$, Duncan test) (Fig. 1).

A statistically significant decrease of cell viability in the HT29 cell line was registered after multiple pre-irradiation with a 0.07-Gy dose compared to a control (II) without pre-irradiation, while low-doses of 0.03 Gy and 0.05 Gy in the same regime induced significant increase of cell viability ( $p<0.05$, Duncan and Tuckey test) (Fig. 1).

Single low-doses pre-irradiation on the MRC5 cell line induced no statistically significant decrease of the cell viability. Multiple low-dose pre-irradiation led to statistically significant decrease of cell viability after $0.03 \mathrm{~Gy}$, while 0.05 Gy induced statistically significant radioadaptive response (Fig. 2).

\section{Discussion}

Radiobiological effects on the HT29 and MRC5 cell lines induced by multiple vs. single low-dose pre-irradiation were compared after a designed irradiation regime of $4 \times 2$ Gy during four days.

Human colorectal carcinoma cells and normal lung fibroblast cells expressed similar radiobiological responses after 0.05 Gy applied as multiple pre-irradiation doses. 


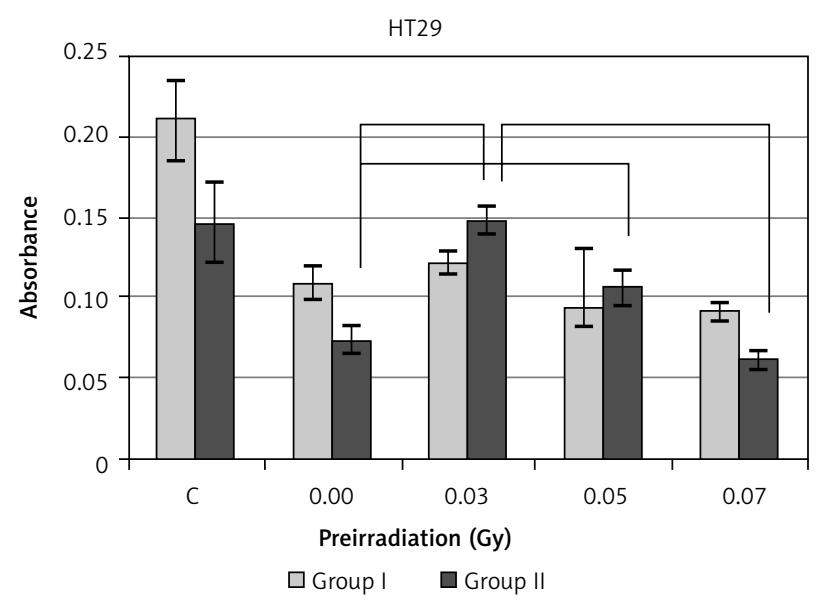

Fig. 1. MTT assay results of multiple vs. single low-dose pre-irradiation on the HT29 cell line. C-non-irradiated cells; cells in both groups received 2 Gy daily during four-day treatment. Cells in group I additionally received a single pre-irradiation dose (0.00-0.07 Gy) 4 hours before irradiation by $2 \mathrm{~Gy}$, while cells in group II received multiple pre-irradiation doses (0.00-0.07 Gy), during four-day treatment, before irradiation by $2 \mathrm{~Gy}$. Solid lines connecting the columns represent statistically significant differences $(p<0.05)$

A low dose of 0.05 Gy applied on the first day induced a non-significant decrease of cell viability in the HT29 cell line, contrary to multiple pre-irradiation, which significantly increased cell viability. In the MRC5 cell line multiple lowdose pre-irradiation with 0.05 Gy led also to a statistically significant increase in cell viability. It can be concluded that the 0.05 Gy low dose caused strong radioadaptive responses in both cell lines.

After a single pre-irradiation dose of $0.03 \mathrm{~Gy}$, a non-significant increase of cell viability was detected by MTT assay in HT29, while multiple pre-irradiation during the same overall treatment time led to an approximately two-fold increase in cell viability. The same low dose in the MRC5 cell line, delivered as single dose, gave a non-significant decrease in cell viability, while in multiple pre-irradiation it led to a statistically significant decrease in cell viability. Multiple 0.03 Gy pre-irradiation dose led to the opposite effect in two cell lines.

A low dose of 0.07 Gy appears to have no effect in a single pre-irradiation regime in both cell lines, while in multiple low-dose pre-irradiation the opposite effect (although non significant) was detected: a decrease in cell viability in the HT29 cell line and an increase in cell viability in the MRC5 cell line.

These facts might indicate that an increase in pre-irradiation dose induces radioadaptive response in the MRC5 cell line and reduces radioadaptive effects in HT29 cells, which could be a subject for further evaluation and possible application in radiotherapy treatment.

In a study designed as a single low-dose pre-irradiation followed by a single challenging dose with 4-hour intervals between doses, a decrease in cell viability was reported for the HT29 cell line [13]. Similar studies gave the same radiobiological response in the human bladder carcinoma cell line after a 0.05 Gy pre-irradiation dose $[13,14]$. In our study, we detected the same response pattern for single application of low dose, while the opposite response was

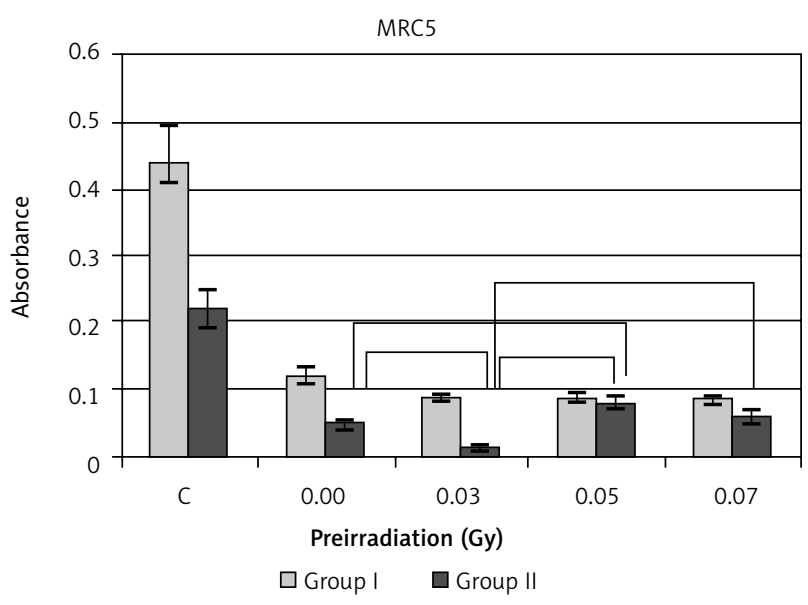

Fig. 2. MTT assay results of multiple vs. single low-dose pre-irradiation on the MRC5 cell line. C-non-irradiated cells; cells in both groups received 2 Gy daily during four-day treatment. Cells in group I additionally received single pre-irradiation dose (0.00-0.07 Gy) 4 hours before irradiation by 2 Gy, while cells in group II received multiple pre-irradiation doses (0.00-0.07 Gy), during four-day treatment, before irradiation by $2 \mathrm{~Gy}$. Solid lines connecting the columns represent statistically significant differences $(p<0.05)$

registered after multiple application of low-dose pre-irradiation. A cell viability increase was previously detected after 0.1 Gy pre-irradiation dose three hours before the challenging dose [19]. Our results indicate a possible increase in cell viability, even though we applied a different regime. We applied the same multiple low-dose pre-irradiation, two hours before daily challenging dose of $2 \mathrm{~Gy}$ during four days, which is a regime more similar to a therapeutic regime in clinical practice. Several authors stated that radioadaptive effects depend on de novo synthesis of proteins occurring after a priming dose, but in a period of 3-24 hours, depending on the cell line, cells return to their basic level of radiosensitivity [3, 19]. In our study we showed that radioadaptive effects can be observed two hours after priming and challenging doses.

However, it seems that level of the pre-irradiation dose plays an important role in induction of different radiobiological effects, since, e.g. in the HT29 cell line 0.03 Gy and $0.07 \mathrm{~Gy}$, whether applied as a single or multiple dose, led to different cell survival. A low dose of $0.03 \mathrm{~Gy}$ in both regimes caused an increase in cell viability, while 0.07 Gy led to a decrease in cell viability in HT29 cells. There is no clear explanation of these phenomena. It is known that p53 responds differently to various priming doses [20]. Activation of p53 may lead to cell cycle arrest or to apoptosis, depending on dose intensity [21]. Colorectal cancer cells may activate protective mechanisms, most probably anti-apoptotic after lower pre-irradiation dose, while a higher pre-irradiation dose could be too challenging for the cell survival mechanisms, and so a cell decrease was detected. In order to prove these speculations, further studies of molecular mechanisms should be done. Differences in radiobiological responses after different priming doses were also detected by others [13], but the exact mechanism is still unknown.

The obtained data prove that the MTT assay represents an adequate approach in evaluating radioadaptive re- 
sponses. Besides the MTT assay, different tests using tetrazolium salts have been used for the estimation of the cell metabolism, based on mitochondrial function [22-24]. Tetrazolium salts are used in cytotoxicity tests and cell proliferation tests since they accept electrons from oxidised substrates or corresponding cofactors, which turns them into 3-(4,5-dimethylthiazol-2-yl)-2,5-diphenyltetrazolium bromide. Formazan is a product of the reaction, a darkblue pigment. The reaction can take place only once in metabolically active cells, so formazan production correlates with the number of viable and metabolically active cells.

In conclusion, multiple low-dose pre-irradiation induced stronger radioadaptive response compared to single lowdose application. Our study design gave several main conclusions: 1) multiple pre-irradiation causes radioadaptive effects in the human colorectal carcinoma cell line for 0.03 Gy and 0.05 Gy, which is also proven for 0.05 Gy in normal foetal lung fibroblasts cells; 2 ) in both single and multiple pre-irradiation regimes 0.07 Gy led to a decrease in cell viability in HT29 and radiosensitivity, while radioresistance was registered in the MRC5 cell line; and 3) radiobiological effects induced by low-dose pre-irradiation were evidently weak during the treatment time, since a single low-dose applied only on the first day expressed no radioadaptive effect. Human colorectal carcinoma cells and normal lung fibroblast cells expressed similar radiobiological responses after 0.05 Gy was applied as multiple pre-irradiation doses. However, the exact mechanisms that underlie the observed effects are still unknown and further effort should be directed towards studies of the molecular mechanisms involved. Nevertheless, the obtained data are promising, especially in the possible application of low-dose pre-irradiation in radiotherapy.

The authors declare no conflict of interest.

This work was financially supported by the Provincial Secretariat for Science and Technological Development, Grant No. 114-451-3943/2013-02 "The effects of low-dose irradiation accompanied by nano-prooxidatives and nano-protectors on human malignant cell lines".

\section{References}

1. Gunderson LL, Tepper LE. Clinical radiation oncology. Elsevier Saunders, Philadelphia 2012.

2. Mothersill C, Seymour RJ, Seymour CB. Increased radiosensitivity in cells of two human cell lines treated with bystander medium from irradiated repair-deficient cells. Radiat Res 2006; 165: 26-34.

3. Joiner MC, Marples B, Lambin P, Short SC, Turesson I. Low-dose hypersensitivity: current status and possible mechanisms. Int J Radiat Oncol Biol Phys 2001; 49: 379-89.

4. Ojima M, Eto H, Ban N, Kai M. Radiation-induced bystander ef fects induce radioadaptive response by low-dose radiation. Radi at Prot Dosimetry 2011; 146: 276-9.

5. Jiang H, Li W, Li X, Wang G. Low-dose radiation induces adaptive response in normal cells, but not in tumor cells: in vitro and in vivo studies. J Radiat Res 2008; 49: 219-30.

6. Hafer K, Iwamoto KS, Scuric Z, Schiestl RH. Adaptive response to gamma radiation in mammalian cells proficient and deficient in components of nucleotide excision repair. Radiat Res 2007; 168: 168-74
7. Coleman MA, Yin E, Peterson LE, Nelson D, Sorensen K, Tucker JD, Wyrobek AJ. Low-dose irradiation alters the transcript profiles of human lymphoblastoid cells including genes associated with cytogenetic radioadaptive response. Radiat Res 2005; 164: 369-82.

8. Venkat S, Apte SK, Chaubey RC, Chauhan PS. Radioadaptive response in human lymphocytes in vitro. J Environ Pathol Toxicol Oncol 2001; 20: 165-75.

9. Zasukhina GD. Radioadaptive response in human cells with different DNA repair activity. Radiats Biol Radioecol 1999; 39: 58-63.

10. Sorensen KJ, Attix CM, Christian AT, Wyrobek AJ, Tucker JD. Adaptive response induction and variation in human lymphoblastoid cell lines. Mutat Res 2002; 519: 15-24.

11. Amundson SA, Do KT, Fornace AJ Jr. Induction of stress genes by low doses of gamma rays. Radiat Res 1999; 152: 225-31.

12. Cramers P, Atanasova P, Vrolijk H, Darroudi F, van Zeeland AA, Huiskamp R, Mullenders LH, Kleinjans JC. Pre-exposure to low doses: modulation of X-ray-induced DNA damage and repair. Radiat Res 2005; $164:$ 383-90.

13. Schwarz SB, Schaffer PM, Kulka U, Ertl-Wagner B, Hell R, Schaffer M. The effect of radio-adaptive doses on HT29 and GM637 cells. Radiat Oncol 2008; 23: 3-12.

14. Schaffer M, Schwarz SB, Kulka U, Busch M, Dühmke E. Adaptive doses of irradiation - an approach to a new therapy concept for bladder cancer? Radiat Environ Biophys 2004; 43: 271-6.

15. Schaffer M, Balandin A, Ertl-Wagner B, et al. Does photofrin II combined with a radio-adaptive dose lead to a synergistic or additive effect after ionising irradiation in vitro? J Can Therapy 2011; 2: 595-600.

16. Bogdanović G, Raletić-Savić J, Marković N. In vitro assays for antitumor-drug screening on human tumor cell lines: dye exclusion test and colorimetric cytotoxicity assay. Arch Oncol 1994; 2: 181-4.

17. Mosmann T. Rapid colorimetric assay for cellular growth and survival: application to proliferation and cytotoxicity assays. J Immunol Methods 1983; 65: 55-63.

18. StatSoft, Inc. (2011). STATISTICA (data analysis software system), version 10. www.statsoft.com.

19. Ryan LA, Seymour CB, Mothersill CE. Investigation of non-linear adaptive responses and split dose recovery induced by ionizing radiation in three human epithelial derived cell lines. Dose Response 2009; 7: 292-306.

20. Enns L, Bogen KT, Wizniak J, Murtha AD, Weinfeld M. Low-dose radiation hypersensitivity is associated with p53-dependent apoptosis. Mol Cancer Res 2004; 2: 557-66.

21. Okazaki R, Ootsuyama A, Norimura T. TP53 and TP53-releted genes associated with protection from apoptosis in the radioadaptive response. Radiat Res 2007; 167: 51-7.

22. Vistica DT, Skehan P, Scudiero D, Monks A, Pittman A, Boyd MR. Tetrazolium-based assays for cellular viability: a critical examination of selected parameters affecting formazan production. Cancer Res 1991; 51: 2515-20.

23. Alley MC, Scudiero DA, Monks A, et al. Feasibility of drug screening with panels of human tumor cell lines using a microculture tetrazolium assay. Cancer Res 1988; 48: 589-601.

24. Wilson AP. Citotoxicity and viability asseys. In: Animal cell culture. Freshney RI (ed.). 1989.

\section{Address for correspondence}

\section{Igor Djan MD}

Institute of Oncology Vojvodina

Put dr Goldmana 4

21104 Sremska Kamenica, Serbia

tel. +381214805624

e-mail: djanigor@yahoo.com

Submitted: 12.11 .2013

Accepted: 25.02 .2014 